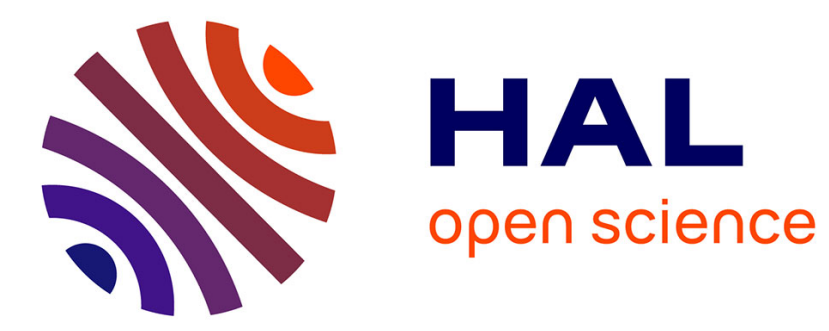

\title{
La paléocarpologie archéologique
}

\author{
Phlippe Marinval, Marie-Pierre Ruas
}

\section{To cite this version:}

Phlippe Marinval, Marie-Pierre Ruas. La paléocarpologie archéologique: Archéobotanique. 1ère partie: les différentes disciplines mises en jeu. Les Nouvelles de l'archéologie, 1985, 18, pp.27 - 32. 10.3406/nda.1985.1360 . hal-02990912

\section{HAL Id: hal-02990912 \\ https://hal.science/hal-02990912}

Submitted on 5 Nov 2020

HAL is a multi-disciplinary open access archive for the deposit and dissemination of scientific research documents, whether they are published or not. The documents may come from teaching and research institutions in France or abroad, or from public or private research centers.
L'archive ouverte pluridisciplinaire HAL, est destinée au dépôt et à la diffusion de documents scientifiques de niveau recherche, publiés ou non, émanant des établissements d'enseignement et de recherche français ou étrangers, des laboratoires publics ou privés. 


\section{La paléocarpologie archéologique}

\section{Philippe Marinval, Marie-Pierre Ruas}

\section{Citer ce document / Cite this document :}

Marinval Philippe, Ruas Marie-Pierre. La paléocarpologie archéologique. In: Les Nouvelles de l'archéologie, n¹8, Hiver 84-85 1985. Archéobotanique. 1ère partie : les différentes disciplines mises en jeu. pp. 27-32;

doi : https://doi.org/10.3406/nda.1985.1360

https://www.persee.fr/doc/nda_0242-7702_1985_num_18_1_1360

Fichier pdf généré le 29/03/2019 


\title{
LA PALEOCARPOLOGIE ARCHEOLOGIQUE
}

\author{
par
}

Philippe MARINVAL \& Marie-Pierre RUAS

La paléocarpologie est l'étude des paléosemences. Celles-ci, retrouvées dans les sites archéologiques, sont constituées par tous les éléments résultant des transformations de 1a fleur (ou de 1'inflorescence), après la fécondation : graines, fruits, pédoncules, téguments, rachis d'épi... carbonisés, minéralisés ou gorgés d'eau. Ces vestiges apparaissent parfois seulement sous forme d'empreinte dans les poteries.

L'intérêt de ce matériel réside dans la compréhension des processus qui lui ont conféré son état final et dans 1 'analyse des relations archéologiques entre ces vestiges et leur contexte (semences / structures, semences / os, etc.).

\section{ETAT DE LA RECHERCHE EN FRANCE}

Bien développée dans les pays voisins (Pays-Bas, Allemagne, Angleterre, par exemple), la paléocarpologie profite depuis peu, en France, d'un courant favorable : ce type d'études prend de plus en plus d'importance dans la recherche archéologique. Cependant la paléocarpologie n'est pas toute jeune en France. Dès 1e XIXème siècle, des études ayant trait à cette discipline débutaient : 1es premiers tra- vaux ont été publiés en 1872 , par J. Le Mire. Si aujourd'hui des programmes s'édifient, des méthodologies s'affrontent, aucun enseignement s'intégrant dans un cursus universitaire comparable à ce qui existe aux Pays-Bas, par exemple, n'a été institué en France (cf. ce volume 1'article de S. Bottema, pp. 75-78); aucun 1aboratoire de paléocarpologie n'est officiellement constitué et pour cause : aucune institution n'a encore accueilli en son sein un spécialiste paléocarpologue (1). Il existe pourtant une forte demande, notamment au sein des écoles, vis-à-vis de l'alimentation et de " 1 a vie quotidienne de 1 'homme préhistorique".

Les études paléocarpologiques sont donc actuellement assurées par des non-professionnels : trois ou quatre étudiants-chercheurs et un ingénieur agronome honoraire (J. Erroux) qui a réalisé $40 \%$ des analyses.

La répartition des études publiées connaît un net déséquilibre diachronique et géographique. Les recherches se sont plutôt orientées vers la connaissance des débuts de I'agriculture, en s'appuyant essentiellement sur les découvertes de céréales (Blé et Orge). Les données concernant les périodes récentes, des Ages des Métaux à 
1 'époque médiévale, sont pratiquement inexistantes. Parallè lement, certaines régions présentent de vastes vides paléobotaniques ; c'est le cas des trois-quarts de 1a France (façade atlantique, Bassin parisien, Massif central...).

\section{APPORTS ET LIMITES DE LA PALEOCARPO- LOGIE.}

L'organigramme proposé (fig. 1) met en évidence 1a répartition des données en deux grands groupes (paléobotanique et paléoethnobotanique). Les informations issues des résultats paléocarpologiques intéressant $1 \mathrm{a}$ paléobotanique, interfèrent avec celles des autres disciplines : anthracologie, palynologie (cf. article de Ch. Leroyer, Nouvelles de l'Archéologie, $\mathrm{n}^{\circ}$ 19, printemps 1985, à paraître). La paléoethnobotanique est plus spécifique à $1 \mathrm{a}$ paléocarpologie dans $1 \mathrm{a}$ mesure où 1 'on étudie des éléments végétaux résultant d'un apport anthropique. A travers ceux-ci, ce sont les activités de 1 'homme que 1 'on tente de saisir.

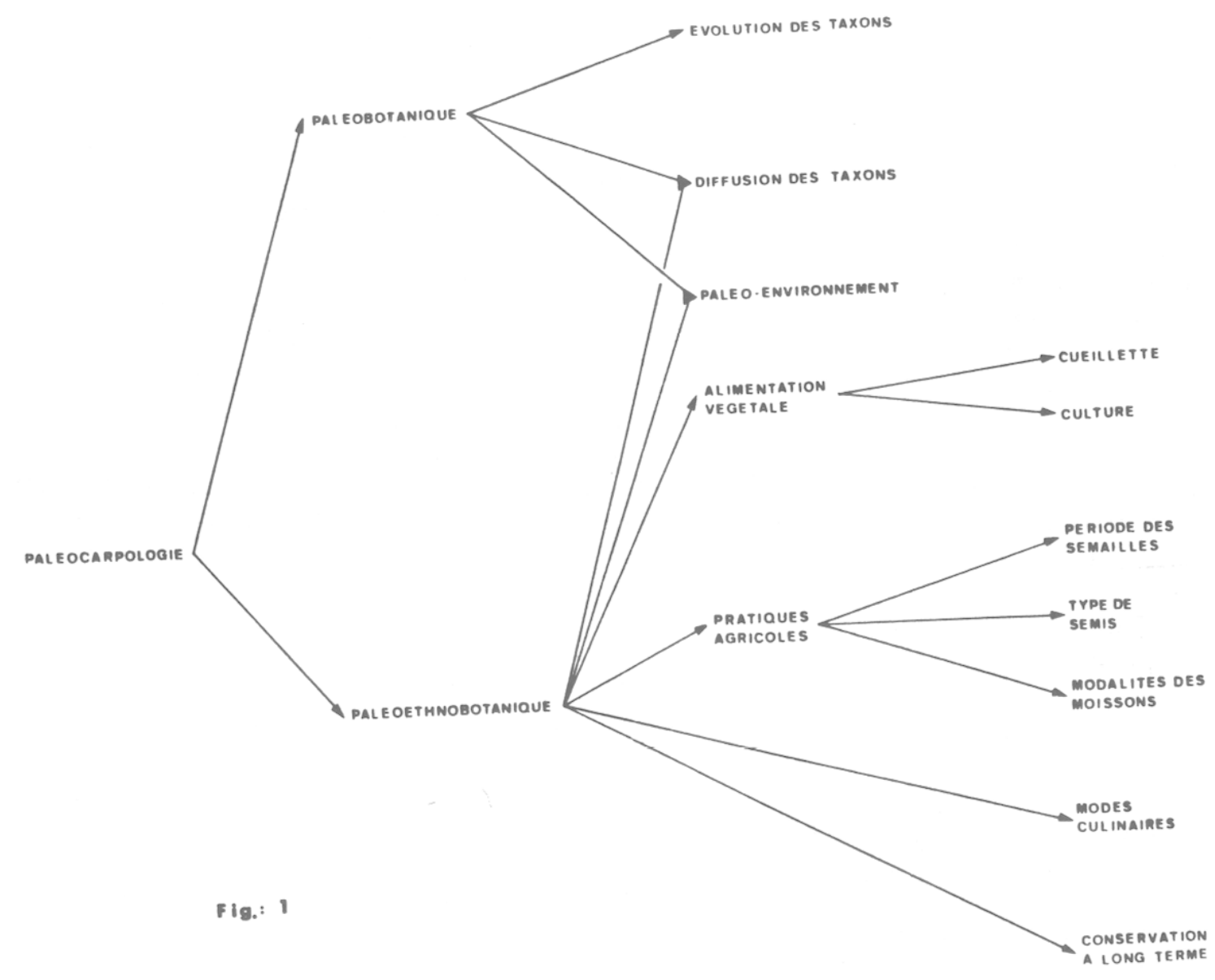


Toutes sortes de semences peuvent apparâิtre dans les "refus" de tamis; nous les réunissons généralement et, de façon arbitraire parfois, en deux ensembles : celui des plantes cultivées et celui des plantes sauvages (2). Cette distinction permet de reconnaître deux activités humaines : 1'agriculture et la cueillette. Toutefois, au sein du groupe des plantes sauvages se côtoient les végétaux cueillis intentionnellement et les plantes couramment qualifiées de "mauvaises herbes" (adventices, rudérales, etc.).

\subsection{Acquisition des données.}

Ainsi, notre travail consiste à tenter de restituer 1 'alimentation végétale sans pour autant négliger les autres données. Cependant, comme dans toute autre spécialité, les résultats dépendent surtout de la méthode et $d u$ soin apporté à la récupération des données.

- ramassage à vue à la fouille : ne sont découverts que les amas ou les concentrations visibles de semences. Cette méthode demeure hasardeuse, inefficace ; et, les vestiges recueillis ne sont aucunement représentatifs.

- Les tamisages : à sec ou sous eau, les tamis habituellement utilisés présentent un maillage inadapté à la dimension du matériel recherché. Nous préconisons des mailles de 1 'ordre de 2 , de 0,5 et de $0,25 \mathrm{~mm}$.

- la flottation. Plusieurs "machines à flottation" fonctionnent actuellement. L'une d'entre elle sera décrite dans le prochain numéro $\left(n^{\circ} 19\right)$. Cette technique, de conception et de réalisation simples, s'emploie plutôt dans le cas de sédiments non constamment humides. Elle assure la séparation presque totale des semences du sédiment enrobant. Le tri en est grandement facilité.

- Les tris. Ceux-ci suivent, le plus sou- vent, I'opération de tamisage. Les fractions grossières $(\geqslant 2 \mathrm{~mm})$ peuvent être triées sur le chantier même. Mais, la diversité des éléments botaniques intéressant le paléocarpologue implique que la personne soit bien exercée et sache ce qu'elle est susceptible de trouver. I1 convient de rappeler que le tri doit être effectué à sec (sauf dans le cas des semences des sites humides). Les fractions fines $(2<x \geqslant 0,25 \mathrm{~mm})$ sont traitées sous une loupe binoculaire.

En ce qui concerne les sites dont les sédiments sont secs, toute technique nécessitant 1 'emploi de 1 'eau exige une humidification lente des prélèvements. L'un d'entre nous (P. Marinval 1984) a mis en évidence la fiabilité de ces différentes méthodes de récupération.

Le diagramme présenté (fig. 2) illustre un test effectué sur cinq échantillons

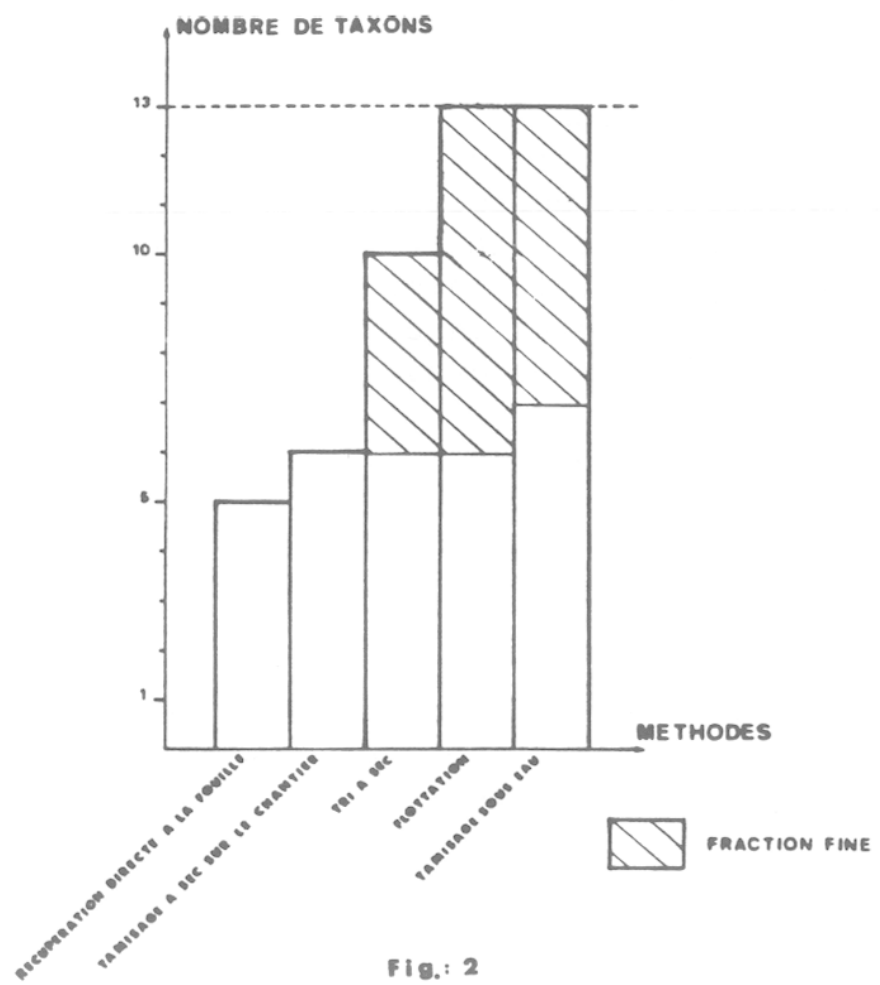


expérimentaux identiques, non archéologiques, dont le contenu en semences carbonisées était connu. I1 s'avère que deux méthodes seulement présentent une réelle fiabilité : le tamisage sous eau et la flottation.

\subsection{L'étude et l'interprétation.}

De par les conditions mêmes de 1 'expérimentation évoquée ci-dessus, il n'a pas été tenu compte de la conservation différentielle des vestiges (facteur non quantifiable). Cet aspect est fondamental au niveau de 1 'interprétation des données archéologiques. La situation de préservation des restes diffère selon qu'il s'agit de sites humides ou non (voir l'article de P. Pétrequin et $K$. Lundströn-Baudais, à paraître dans le $\mathrm{n}^{\circ} 19$ des Nouvelles de l'Archéologie). Dans le deuxième cas, seuls sont conservés les macro-restes végétaux carbonisés ; d'autres fossilisations plus rares peuvent cependant survenir. La carbonisation soumet les éléments initialement présents à une sélection qui s'effectue en fonction de la constitution biochimique des tissus et de la dimension du matériel. En conséquence, il paraît très aléatoire d'établir des calculs de pourcentages sur le nombre des individus ou leur poids, dans le cadre de paléosemences dispersées dans une couche.

Les travaux de K. Lundström-Baudais (1984) sur le site de Clairvaux-les-Lacs (Jura) le démontrent. Ce gisement d'ambiance humide recèle à la fois des semences carbonisées et non carbonisées.

La figure 3 représente les différents modes de décompte du nombre de restes (exprimés en pourcentages) effectués sur sept plantes cultivées.

Ainsi, sont pris en considération :

- A : seulement les grains carbonisés (graines et grains de céréales - caryopses - ).
- B : 1a totalité des grains (carbonisés et non carbonisés).

- C : dans le cas des céréales, l'évaluation du nombre de grains à partir des restes de battage.

- D : le poids des semences.

\begin{tabular}{|c|c|c|c|c|}
\hline ESPEECES & A & B & C & D \\
\hline Triticum dicoccum & $2 \%$ & \multirow{4}{*}{$8 \%$} & $40 \%$ & $60^{\circ}$ \\
\hline Triticum monococeum & + & & . 10 & \\
\hline Triticum aestivum & $3 \%$ & & $12 \%$ & $21 \%$ \\
\hline Hordeum valgare & $89 \%$ & & $8 \%$ & $15 \%$ \\
\hline Papaver somniferum & $3 \%$ & $79 \%$ & $34 \%$ & + \\
\hline Linum usitatissimum & $3 \%$ & $13 \%$ & $6 \%$ & $1 \%$ \\
\hline Pisum sativum & + & + & + & $1 \%$ \\
\hline
\end{tabular}

(d'après K. Lundström-Baudais)

Fig.: 3

Ils permettent de constater que les valeurs sont différentes d'un cas à l'autre, au point que les proportions relatives peuvent s'inverser (exemple : le cas A correspondrait à la situation d'un site à sédiments secs, le cas $B$ celle d'un site à sédiments humides).

Si la quantification du nombre de restes de paléo-semences demeure sujette à caution dans le cadre d'un seul gisement, elle ne peut être totalement bannie. La comparaison des calculs de proportion relative à des taxons sur de nombreux sites devrait, à long terme, permettre d'établir une synthèse paléo-économique.

Jusqu'à présent, 1 'interprétation statistique des pourcentages des paléo-semences, en ce qui concerne la composition des milieux clos (3) s'avère plus rigoureuse. 
Ces ensembles étant ponctuels dans le temps, les effets parasites, tels les éléments intrusifs, n'interviennent pratiquement pas.

L'analyse des contenus de ces milieux peut mettre en évidence le degré d'homogénéité de 1 'échantillon : elle rend possible une meilleure approche des pratiques agricoles. Ainsi, la présence concomitante de plusieurs taxons (céréales et légumineuses, etc.) peut procéder d'un mélange, lors de 1 'entreposage des denrées ou refléter une culture de type méteil (semis simultané de genres et/ou d'espèces différentes). De même 1'absence, la présence et la nature des plantes adventices, l'existence d'un calibrage des grains, donnent de précieuses indications sur les périodes de semailles, les types de semis, les modalités des moissons... La relation entre la structure archéologique (grenier, si1o, etc.) et son contenu livre des informations sur la conservation des grains à long terme.

L'utilisation des denrées provenant d'une cueillette ou d'une culture prend divers aspects selon le mode de consommation choisi. L'état du vestige et les découvertes de reliquats de préparations culinaires (fragments de galettes, bouillies, etc.) permettent d'émettre des hypothèses sur les modes culinaires, tout en tenant compte des facteurs contextuels du matériel (four par exemple).

\section{CONCLUSION}

La paléocarpologie s'intègre donc dans le cadre d'une approche palethnologique et paléoécologique.

Les échanges permanents avec les ethnobotanistes (qui travaillent sur les périodes sub-actuelles et contemporaines) permettent de connaître les potentialités d'utilisation de la flore sauvage et de mieux comprendre la structuration de 1 'environnement naturel.

Cependant, dans I'interprétation des données archéologiques, il est impératif de tenir compte des facteurs taphonomiques et d'établir des relations interdisciplinaires notamment avec les archéozoologues.

N'oublions pas que les problèmes de subsistance sont une des composantes essentielles de la survie de tout organisme vivant.

\author{
P. M. \\ Laboratoire de Paléo- \\ botanique (U.A. 275), \\ Musée de 1 'Homme, \\ Paris. \\ M. -P. R. \\ 14 rue Linné \\ 75005 Paris.
}

\section{NOTES}

(1) On peut cependant espérer, en 1985, 1 'amélioration de cette situation avec un fléchage de poste, au CNRS, pour 1'étude des macro-restes végétaux au Laboratoire de Chrono-Ecologie de Besançon.

(2) Il est parfois délicat d'opter de façon rigoureuse pour 1 'une ou 1 'autre catégorie, dans le cas de certains végétaux qui peuvent être considérés domme des plantes de semi-culture - procédé qui permet une meilleure croissance de l'individu sauvage par l'élimination des plantes concurrentes ou par transplantation de 1 'individu autour de 1 'habitat : arbres fruitiers (Noisetiers, Merisiers, Pommiers...) - ou des plantes herbacées (Panais, Légumineuses, etc.).

(3) Il s'agit d'ensembles bien définis 
dans I'espace et constitués d'éléments synchrones : récipients, greniers, silos, etc., remplis de paléo-semences.

\section{REFERENCES}

LE MIRE (J.), 1872.-Découvertes d'une station lacustre de l'Age de la pierre polie dans le lac de Clairvaux. Recueil de 1 'Académie de Besançon.- 52 p., 6 pl.

LUNDSTROM-BAUDAIS (K.), 1984.- Palaeo-ethnobotanical Investigation of Plant Remains from a Neolithic Lakeshore Site in France:
Clairvaux, Station III, pp. 293-305, in : W. van ZEIST et W.A. CASPARIE (sous $1 a$ dir. de), Plants and Ancient Man.- Groningen.

MARINVAL (Ph.), 1984.- Recherches expérimentales sur l'acquisition des données en paléocarpologie. - Mémoire de DEA de 1'EHESS, 46 p., 6 fig., 8 tabl., Paris.

carpologie : ses apports ; les problèmes d'acquisition des données et 1 'avancement de 1 a recherche en France.- Actes du Colloque : Journées de palynologie archéologique, 25, 26 et 27 janvier 1984.- Valbonne, Centre de recherche archéologique. 